\title{
Transverse myelitis following combined spinal-epidural anesthesia
}

\author{
Jung Ho Seok, Youn Hee Lim, Seung Hoon Woo, and Jun Heum Yon \\ Department of Anesthesiology and Pain Medicine, Sanggye Paik Hospital, College of Medicine, Inje University, Seoul, Korea
}

Transverse myelitis is an inflammatory or demyelinating disease of the spinal cord, which causes bilateral hypoesthesia and motor weakness, a decrease in deep tendon reflex spasticity of the extremities, and urination and defecation problems in the areas lower than affected spinal segment, without any observation of spinal cord injury, spinal lesion, or tumor, and no observation of pressure on the spinal cord [1]. We report our experience of transverse myelitis that occurred after spinalepidural combined anesthesia for caesarian section with a literature review. A 37-year-old pregnant woman (height $162 \mathrm{~cm}$, weight $72 \mathrm{~kg}$ ) with no specific medical history and no abnormalities was performed spinal-epidural combined anesthesia in the L 3-4 epidural space with intrathecal administration of 5\% bupivacaine $7 \mathrm{mg}$ for caesarean delivery at $39+1$ weeks. Vital signs were stable with no specific event during this procedure and surgery. Fifteen minutes before the end of the surgery, fentanyl $50 \mu \mathrm{g}$ and $1 \%$ lidocaine $40 \mathrm{mg}$ were injected into the epidural space with N/S $2 \mathrm{ml}$ for post-operative analgesia. For patient controlled analgesia, fentanyl $350 \mu \mathrm{g}+0.5 \%$ chirocaine $50 \mathrm{mg}+\mathrm{N} / \mathrm{S} 83 \mathrm{ml}$ were combined and connected to the epidural catheter with the following settings: basal rate $2 \mathrm{ml} /$ hr, bolus $1 \mathrm{ml} / \mathrm{hr}$, and lockout time $30 \mathrm{~min}$. Thirty minutes after transported into the recovery room, the anesthesia was up to T12, and the patient was moved to a general ward after confirmation of stable vital signs. Two days after surgery, the epidural catheter was removed. But the patient continuously complained of hypoesthesia and motor weakness in the left lower extremities. The condition gradually worsened to both sides so a lumbar and thoracic MRI was performed, but no abnormalities were found. Four days after surgery, WBC count was $30 \mathrm{cell} / \mathrm{mm}^{3}$ and protein was $91 \mathrm{mg} / \mathrm{dl}$ in the cerebrospinal fluid examination. Except that, abnormal finding was not seen. The patient was IV injected with methylprednisolone 1,000 mg as a steroid pulse therapy for 5 days for suspicion of transverse myelitis. Five days after surgery, in the somatosensory evoked potential examination, bilateral peripheral conduction disturbance was observed in the posterior tibial nerve. The lumbar magnetic resonance imaging performed 7 days after surgery was normal like before, and brain MRI was normal. Ten days after surgery, motor power of lower extremities slightly increased. Thus, mobility was recovered and prednisolone $40 \mathrm{mg}$ was orally administered from that day. Twenty-two days after surgery, an electrophysiology test's results showed radiculopathy in the L3-S1 region on both sides. Steroid therapy was stopped and walking practice was performed along with physical therapy. Six months after surgery, the right side displayed nearly normal motor function. The left side showed grade III hip joint flexion and grade IV extension, grade IV knee joint flexion and extension, and grade II ankle joint dorsiflexion and grade III extension. Approximately 7 months after the surgery, the patient was discharged. The prevalence of transverse myelitis is 4.6 per million in the United States, and mostly develops in young adults and middle-aged people regardless sex. Most of this disease is idiopathic but it can accompany vascular malformation or autoimmune disease such as multiple sclerosis, systemic lupus erythematosis. Also myelitis symptoms developed from preceding virus infections such as EbsteinBarr virus, cytomegalovirus was reported. As seen in our case,

Corresponding author: Youn Hee Lim, M.D., Department of Anesthesiology and Pain Medicine, Sanggye Paik Hospital, College of Medicine, Inje University, 761-1, Sanggye 7-dong, Nowon-gu, Seoul 139-707, Korea. Tel: 82-2-950-1173, Fax: 82-2-950-1323, E-mail: painfree@paik.ac.kr (c) This is an open-access article distributed under the terms of the Creative Commons Attribution Non-Commercial License (http:// creativecommons.org/licenses/by-nc/3.0/), which permits unrestricted non-commercial use, distribution, and reproduction in any medium, provided the original work is properly cited. 
transverse myelitis can develop in spinal anesthesia. Paresthesia from spinal cord injury can occur not only from needle insertion, but also from the toxicity of the regional anesthesia, secondary irritation and edema from injection, hematoma, occlusion of spinal cord artery, and a state of hypoperfusion [2]. Contrary to the case reported by Teh et al. [3] where patient's recovery time was within 24 hours, our case had a very long recovery period. Since the regional anesthesia used was not a high dose, it is unreasonable to contend that the patient developed regional anesthesia induced transverse myelitis. Also Jha and Kumar [4] reported transverse myelitis occurred after the patient experienced pain like severe electric shock when inserting the needle during spinal anesthesia. However our patient did not complain of pain and no observations of pressure such as a hematoma was found in the MRI. When spinal anesthesia is performed on patients in sedation state they become insensitive to pain, and paresthesia can be developed from spinal cord damage. However, the patient in our case did not receive any sedatives or analgesics before and during spinal anesthesia so this possibility is low. Nevertheless, it cannot be ruled out that the patient had insufficient information about spinal anesthesia and ignored any pain she felt. Approximately half of the patients with transverse myelitis complain of pain; however, there could be cases like ours where the patient only complains of muscle weakness and hypoesthesia without pain. Chronic neuropathic pain can also occur in some patients. The prognosis is known to be relatively good, but in severe cases, permanent physical disabilities can remain or even lead to death. Steroid IV injection is used as the primary treatment for acute central nervous system demyelinating disease, and antidepressants, anticonvulsants, analgesics, etc. are used too. When such medical treatment is ineffective, interventions such as nerve blocks can be attempted but the effects are insignificant. Although the physiological mechanism is not clear, there are reports that show spinal cord stimulation has been effective in treating pain. Most of the causes of transverse myelitis are idiopathic and it can occur without evidence of hematoma or infection after combined spinal-epidural anesthesia as in our case.

\section{References}

1. Berman M, Feldman S, Alter M, Zilber N, Kahana E. Acute transverse myelitis: incidence and etiologic considerations. Neurology 1981; 31: 966-71.

2. Houten JK, Errico TJ. Paraplegia after lumbosacral nerve root block: report of three cases. Spine J 2002; 2: 70-5.

3. Teh HS, Fadilah SA, Leong CF. Transverse myelopathy following intrathecal administration of chemotherapy. Singapore Med J 2007; 48: e46-9.

4. Jha S, Kumar R. Transverse myelitis following spinal anesthesia. Neurol India 2006; 54: 425-7. 